\title{
Effect of Loading Wave Profile on Hydrodynamic Void Collapse in Detonation Initiation
}

\author{
A.B. Swantek*, R.K. Shukla ${ }^{\dagger}$, and J. M. Austin ${ }^{\ddagger}$ \\ University of Illinois, Urbana-Champaign, 61801
}

\begin{abstract}
We experimentally and numerically investigate void collapse as a mechanism for detonation initiation in porous energetic materials under a stress-wave loading condition, representative of accidental mechanical insult. In contrast to the step loading of a shock, a stress wave induces a ramp loading, where length scales of the wave may be comparable to the void size. Using an inert and transparent polymer material, we decouple the reactive and material aspects of void collapse, and focus instead on the hydrodynamic process of interactive void collapse. Diagnostic techniques include high speed shadowgraph movies of the collapsing voids and particle image velocimetry in the surrounding material. Two dimensional finite volume simulations compare the interaction of a single void undergoing ramp and shock wave loading. Voids exhibit asymmetric collapse, with formation of a high speed jet that originates from proximal wall of the void. Data obtained, including internal volume histories and collapse times of current experiments and simulations, are reported and compared with shock-induced cavity collapse data from the literature.
\end{abstract}

\section{Introduction}

The formation of regions of localized energy release, or hotspots, is critical to detonation initiation in heterogeneous energetic materials. Hotspots are formed due to the interaction of the loading wave with microscale and molecular-scale material heterogeneities through processes such as debonding, microcracking, and shear-banding. ${ }^{1}$ One mechanism by which hotspots can be created is the collapse of voids, which typically form 1-2\% of an ideal plastic-bonded explosive. If the chemical and mechanical energy release can couple and overcome dissipation, a detonation is initiated. ${ }^{2}$ Understanding the dominant mechanisms of hotspot formation is critical for determining ignition performance, as well as establishing safe handling procedures.

There is an extensive literature examining the collapse of voids or bubbles under shock loading which, for energetic materials, is applicable in performance (high-impact velocity) applications. In the present work, we instead examine a slower loading wave with a ramped profile, typical of an accidental (low-impact velocity) scenario in which a stress wave is induced in the device casing. The length scales of the loading wave profile are comparable with the void length scales, potentially resulting in a strongly coupled interaction under stress wave loading.

Numerous researchers ${ }^{3-10}$ have examined energy localization mechanisms during the collapse of a single void, identifying several relevant mechanisms: shock focusing, adiabatic gas compression, jetting, hydrodynamic and viscoplastic work. A Reynolds number Re based on the void size can be defined as

$$
R e=\delta_{o} \sqrt{\rho p_{s}} / \mu
$$

where $\delta_{o}$ is the critical void size, $p_{s}$ is the loading wave strength, and $\rho$ and $\mu$ are the density and viscosity in the surrounding media. ${ }^{5}$ For small Reynolds numbers corresponding to small void diameters, the collapse can be considered in a viscous regime. For high Reynolds numbers and larger voids, the collapse is in the hydrodynamic regime. ${ }^{5,10}$ In the present study, we focus on the hydrodynamic processes that occur as a single void collapses. In the hydrodynamic collapse regime, loading is typically parametrized by the pressure

\footnotetext{
${ }^{*}$ Graduate Student, Department of Aerospace Engineering, Student Member AIAA

${ }^{\dagger}$ Postdoc, Computational Science and Engineering

¥Assistant Professor, Department of Aerospace Engineering, Senior Member AIAA
} 
across the shock wave. In the case of a distributed loading wave, the length scale associated with the pressure rise may become an additional parameter affecting void collapse history.

We carry out dynamic experiments in a model setup similar to that pioneered by Dear et al. ${ }^{11}$ for a different loading condition. Gas-filled voids in a thin sheet of polymer material are sandwiched between two optically transparent plates, and subjected to a planar stress wave induced by a gas gun and striker plate. High-speed shadowgraph images are used to track the precursor wave and void interfaces as a function of time. In addition, full velocity field data during passage of the loading wave and collapse of the void array are obtained via particle image velocimetry (PIV) measurements. Inviscid numerical simulations of single cavity collapse are carried out at the experimental conditions. The effect of loading wave length scale and profile is examined, and results are presented in the context of the existing shock-induced collapse literature.

\section{I.A. Background}

In addition to their critical role in condensed phase detonation initiation, shock-cavity interactions occur in a wide range of applications. As a result, extensive experimental and computational work has been performed for the collapse of a single void in reactive and non-reactive media under shock loading, typically parametrized by the pressure ratio. Ding \& Gracewski ${ }^{12}$ performed a two-dimensional computational study of a gas cavity exposed to shocks of various strength. A weak shock was defined as one which resulted in symmetric cavity collapse, which was observed to occur if the pressure ratio across the shock wave was less than 300. A strong shock was defined as one which resulted in asymmetric cavity collapse, which was observed to occur if the pressure ratio was greater than 5000. Intermediate cases were not investigated in this study. For the strong shock case, the proximal interface rapidly coalesces into a jet which impinges upon the distal interface, asymmetrically collapsing the cavity.

The formation of an internal, high-speed jet was experimentally observed by Dear et al. ${ }^{11}$ and Bourne \& Field, ${ }^{13}$ in study of a $0.26 \mathrm{GPa}$ shock interaction with 3 to $12 \mathrm{~mm}$ diameter cylindrical voids in a $12 \%$ gelatinwater mixture. The jet tip was observed to propagate at roughly twice the post-shock particle velocity, which is in agreement with the acoustic approximation as well as the results of Ding \& Gracewski. ${ }^{12}$ Additionally, the authors estimate the jet velocity could increase another factor of 1.5 due to nonlinear effects. A summary of normalized volume versus time data was presented and compared with existing results from the literature. Bourne \& Field ${ }^{13}$ found a linear curve fit to these data.

Strong shocks with pressure ratios up to $3.49 \mathrm{GPa}$ were also investigated in a related study. ${ }^{14}$ In this case, the internal jet was observed to propagate through the void ahead of the incident shock with a velocity of 7.5 times that of the particle velocity in the medium. The impact of the jet on the distal wall created a shock wave which overtook the incident loading wave. The high-speed jet and the consequent "water-hammer" impact on the material downstream of the void has been examined numerically and experimentally by numerous researchers. For example, in an inert material with constitutive properties of HMX, Menikoff ${ }^{9}$ observed that under strong shock loading, the internal jet propagated at velocities comparable to the shock velocity and its impact on the distal interface generated a Mach reflection in the lead shock.

Ball et $a l .{ }^{15}$ simulated a cylindrical, $6 \mathrm{~mm}$ diameter, air-filled void in water for comparison with Bourne \& Field ${ }^{13}$ experiments. When exposed to a $1.9 \mathrm{GPa}$ shock, the void was found to collapse asymmetrically and form a high speed jet. The impact of this jet on the distal wall created a 4.7 GPa blast wave which propagated into the downstream media. Bourne \& Milne ${ }^{16}$ discuss the importance of the jets in the ignition process. Experimental and numerical results indicate that a reaction zone is always formed ahead of the lead shock. When comparing simulations, they saw that in the reactive case, the shock is accelerated in comparison with the inert case, illustrating the importance of coupling between the fluid dynamics and the chemical energy release. Ball et al. ${ }^{15}$ and Turangan et al. ${ }^{17}$ report the normalized volume versus time behavior observed in their simulations of cylindrical cavity collapse. Their data exhibits nonlinear behavior and deviates from the linear experimental data fit of Bourne \& Field. ${ }^{13}$ Johnsen \& Colonius ${ }^{18}$ simulated the collapse of a spherical air bubble in water, examining shock-induced collapse and Rayleigh collapse in a free field and near a rigid boundary. A range of pressure ratios were examined. Normalized volume versus time data show a nonlinear collapse history.

In the above studies, the loading condition is a shock, such as would be induced by high velocity projectile impact. In the present study, similar to our previous study, ${ }^{19}$ we instead examine a stress wave loading condition which, in an energetic material, might be induced in the casing through accidental mechanical insult. The length scales of the loading wave profile are chosen to be comparable to the void size. Diagnostic techniques include high speed shadowgraph movies of void collapse and particle image velocimetry measure- 
ments in the surrounding media. We compare our current experimental and numerical results for the single void collapse to quantify the effects of stress wave versus shock loading.

\section{Experimental Setup}

The gas gun used in this experiment accelerates a hardened maraging steel projectile $(\phi=25 \mathrm{~mm})$ down a $2.1 \mathrm{~m}$ barrel to a velocity of $27.0 \pm 0.3 \mathrm{~m} / \mathrm{s}$ by means of a reservoir pressurized to 15 psi with air. Near the end of the gun barrel, three sets of infrared emitters and detectors are positioned to measure the projectile velocity, and subsequently serve as an experimental trigger.

The test sample is made by sandwiching a thin $(1.6 \mathrm{~mm})$ sheet of inert gelatinous material between two optically accessible plates. A mixture of agarose and glycerol gradient buffer (GGB) is set as a gelatinous sheet into which $3 \mathrm{~mm}$ cylindrical, air-filled voids can easily be introduced. The gel contains $5 \%$ agarose by volume in GGB. The mold, shown in Fig. 1a, is made from polymethyl methacrylate (PMMA), which in turn serves as windows for optical access. An aluminum striker plate used to create the loading condition of interest. The usable gel test sample is $85 \mathrm{~mm}$ x $30 \mathrm{~mm}$ x $1.6 \mathrm{~mm}$. The projectile impacts the striker plate, introducing a stress wave which then produces the loading condition in the gel material. The spacing between the striker plate and the center of void is $5 \mathrm{~mm}$. Inside the test section, the sample is placed without restraint immediately downstream of the exit of the barrel. A schematic of the gas gun and test section is shown in Fig. 1b.

The material properties of agarose-GGB gel were obtained from a separate series of experiments. In order to determine the Young's modulus, a quasi-static compression test using a MTS Alliance RT/30 load frame was conducted, yielding an average Young's Modulus value of $38.2 \pm 4.0 \mathrm{kPa}$. This matched well with results obtained for gelatin. ${ }^{20}$ The sound speed of the Agarose-GGB gel was obtained using a JSR Ultrasonics PR35 Ultrasonic Pulser/Receiver, resulting in an average of $1500 \pm 10 \mathrm{~m} / \mathrm{s}$.

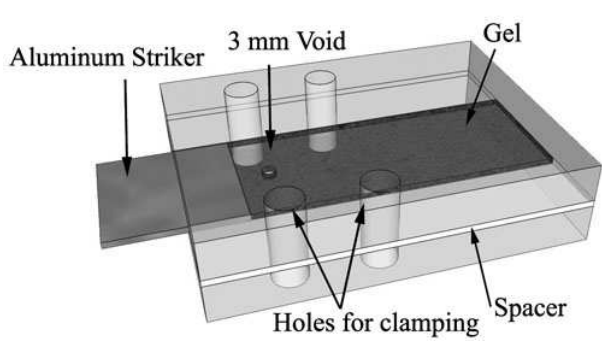

(a)

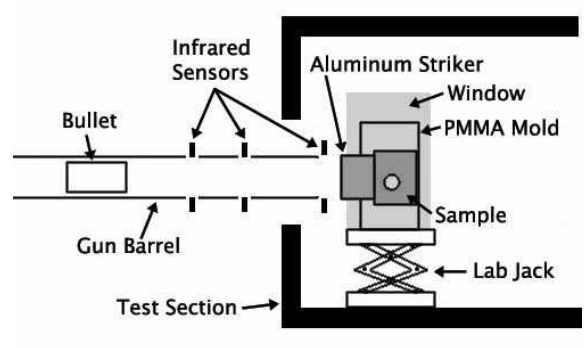

(b)

Figure 1: a) Sketch of mold used to create samples of gelatinous material with voids. The projectile impacts the striker plate which transmits the loading waves to the sample. b) Schematic of gas gun and test section showing the projectile, projectile velocity measurement and diagnostic trigger sensors, and sample location.

A Utah Image Systems high-speed, 80 frame, rotating mirror camera is used to acquire time-resolved shadowgraph images of the void collapse process. A 2 watt $532 \mathrm{~nm}$ solid state continuous wave laser provided illumination for the experiment, with a $200 \mathrm{~ns}$ exposure time, controlled by an acousto-optic modulator. The light is collimated $(\mathrm{f}=150 \mathrm{~mm}$ ) to a $20 \mathrm{~mm}$ field of view. The inter-frame time can be varied and is set to $1.5 \mu \mathrm{s}$. Error bars on shadowgraph movie data are $\pm 0.23 \mathrm{~mm}$ in position and $\pm 0.75 \mu$ s. These images not only serve to track the void interfaces with time, but can provide void area (and subsequently volume assuming a constant sample thickness of $1.6 \mathrm{~mm}$ ) as a function of time during the collapse.

Particle image velocimetry (PIV) measurements are conducted using a two-color (625 nm and $530 \mathrm{~nm})$, single-frame technique. Hollow glass spheres, approximately $20 \mu \mathrm{m}$ in diameter, are introduced into the gel before it is poured into the mold. The PIV setup, Fig. 2, consists of a pair of fast response, high energy LEDs by Innovative Scientific Solutions Inc. (ISSI), which after triggering, fire sequentially (each for $500 \mathrm{~ns}$ ) with a known delay of $3 \mu \mathrm{s}$. Light from the two LEDs passes through a prism and illuminates a 30mm field of view in the gel. A Nikon D50 camera is triggered manually. The raw data is analyzed using dPIV 2.1 (ISSI) software. The resolution of the velocity measurements is $2 \mathrm{~m} / \mathrm{s}$. Similar experimental techniques have been employed in the study of a comparable gel material, polydimethlysiloxane (PDMS), as dynamic witness plate for the characterization of exploding bridge wire detonators. ${ }^{21,22}$ The transparent gel allowed optical 


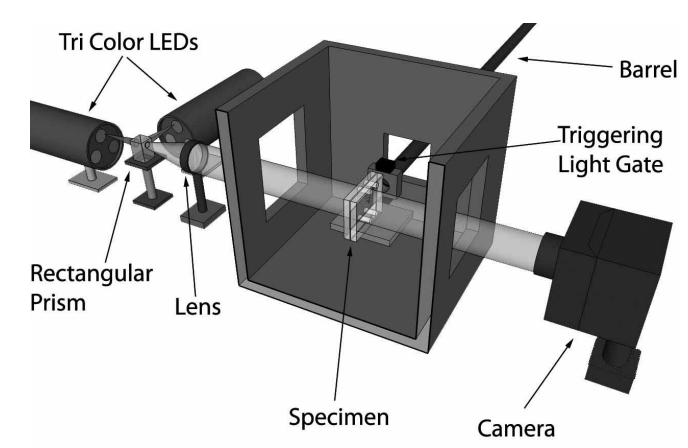

Figure 2: Schematic of the two-color, single-frame particle image velocimetry experiment.

access for schlieren visualization of the blast wave, as well as PIV measurements in the post-shock fluid.

\section{Numerical flow solver}

In order to simplify analysis we neglect the elastoplastic character of the loading wave and study the interaction of a collapsing void with a ramp loading profile in an inviscid fluid. This can provide only a crude model for void collapse in agarose gel, however, such interaction does not appear to have been studied previously. Extension of simulations employed here to a viscoelastoplastic model is a non-trivial task and will be considered in future. Here, we consider compressible multiphase two-dimensional flows which are governed by a system of nonlinear hyperbolic conservation laws given by

$$
\begin{aligned}
\frac{\partial \rho}{\partial t}+\nabla \cdot(\rho \mathbf{u}) & =0, \\
\frac{\partial \rho \mathbf{u}}{\partial t}+\nabla \cdot(\rho \mathbf{u u})+\nabla p & =0, \\
\frac{\partial E}{\partial t}+\nabla \cdot[\mathbf{u}(E+p)] & =0,
\end{aligned}
$$

where $\rho$ is the density, $p$ is the pressure, $\mathbf{u}$ is the velocity, and $E$ is the total energy (kinetic plus internal). The liquid thermodynamics are modeled via a stiffened equation of state,

$$
p=\rho(\gamma-1) e-\gamma p_{\infty},
$$

where $e$ is the mass-specific internal energy. The model parameters in (5) were selected to be $p_{\infty}=3.4 \times 10^{8} \mathrm{~Pa}$ and $\gamma=7$, which provides an approximate model for agarose. The void contents are modeled by the ideal gas equation of state for air: (5) with $p_{\infty}=0$ and $\gamma=1.4$. The effects of viscosity, heat conduction and mass diffusion are not accounted for in this model as the collapse and jetting are expected to occur too quickly for any significant phase change or thermal transfer between the void and surrounding gel.

We discretize equations (2) through (5) using a finite volume method which utilizes a TVD reconstruction with a minmod limiter ${ }^{23}$ and an HLLC ${ }^{24}$ approximate Riemann solver. These are standard techniques for single-phase gas dynamics calculations involving shocks. In order to track the two fluids in our system (the gas and surrounding fluid), we also transport a phase field, which is used to demark the different regions that the two fluids occupy: $\phi=1$ in the void and is 0 elsewhere. A wide class of so-called level-set or phase-field schemes model the interfaces, which in reality are molecularly thin, with a mesh-resolved though narrow continuous variation of $\phi$ between its extreme values on either side. With such a 'smeared' interface model, the transport of $\phi$ is governed by

$$
\frac{\partial \phi}{\partial t}+\mathbf{u} \cdot \nabla \phi=0
$$

Numerical diffusion in general will further smear these interfaces in time, which greatly degrades the quality of long-time solution. However, we have designed special terms based upon initialization of the phase field using a tanh profile diffused over a few grid cells and keeping this profile fixed as it advects during the simulation. ${ }^{25,26}$ When coupled into the overall numerical scheme this preserves the sharpness of the $\phi$ 
representation of the material boundaries. These and all the other details of the the scheme are presented in full elsewhere. ${ }^{27}$ A third-order accurate TVD Runge-Kutta method ${ }^{28}$ is used for time advancement.

The computational domain extends $22.5 \mathrm{~mm}$ in $x$ and out to $11.25 \mathrm{~mm}$ in $y$ and was discretized by $1000 \times 500$ mesh points in these two directions, respectively. For all the results reported, no appreciable change was observed for a $500 \times 250$ mesh calculation. The $3 \mathrm{~mm}$ diameter void is impacted by a ramped wave which achieves a pressure ratio of 421 over a length of $9.9 \mathrm{~mm}$ and is approximated using acoustic relationships for small amplitude disturbances. Simulations were run with time step $\Delta t$ adjusted to fix the CFL number: $\Delta t(c+|u|)_{\max } / h=0.3$, where $h=\Delta x=\Delta y$ is the mesh spacing.

\section{Results}

\section{IV.A. Characterizing the Stress Wave Loading Condition}

We first quantify the elastic-plastic loading condition in the gel material without voids. Several investigators have reported on the development of elastic plastic waves in HMX as a function of impact velocity. ${ }^{29-31}$ Figure 3a presents data from Jaramillo et $a .^{29}$ which shows an intermediate range of piston speeds where both an elastic and plastic wave are present, before steepening to a shock. In the present work, the impact of the steel projective on the aluminum striker produces an elastic precursor followed by a plastic wave which induces a ramped velocity profile up to the projectile velocity $U_{p}$, Fig. $3 \mathrm{~b}$. Thus, our loading condition resides in the intermediate regime. The length scales of the ramped velocity wave profile are comparable to the void diameter, potentially resulting in dynamic interaction between the propagating loading wave and the collapsing void.

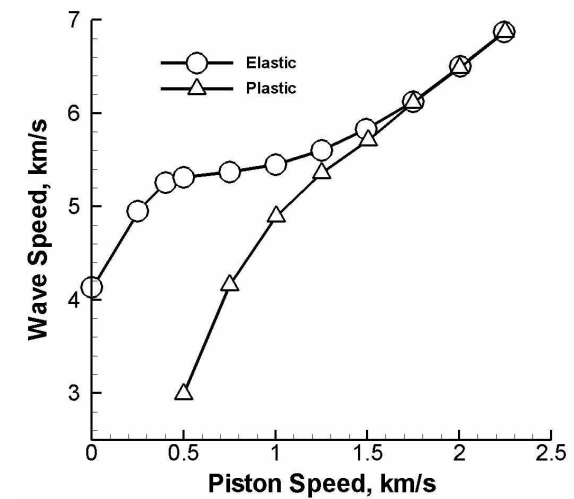

(a)

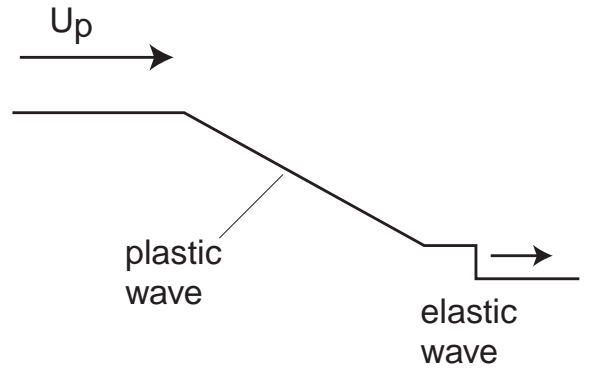

(b)

Figure 3: a) Wave velocity versus piston velocity in HMX, from. ${ }^{29}$ The present experiments examine a lower impact velocity which produces an elastic-plastic wave in the striker plate. b) Representation of an elastic-plastic wave.

The pressure pulse resulting from the elastic precursor wave is tracked using high speed shadowgraph movies. These data are shown on an $x-t$ diagram in Fig. 4 . The pulse is measured to propagate at $1580 \pm 38 \mathrm{~m} / \mathrm{s}$. From the PIV measurements, we observe the precursor wave induces no detectable velocity field, Fig. 5 .

The ramped velocity profile, generated by the plastic wave, contrasts the discontinuous velocity profile which results from shock wave loading. To characterize the loading condition, we measure velocity profiles at several horizontal cross-sections in the gel from a single PIV image, Fig. 5a. These data are taken approximately $11 \mu \mathrm{s}$ after the precursor enters the gel. The velocity profile increases monotonically to the projectile velocity. The wave front is reasonably planar with similar velocity profiles at each cross-section examined. The evolution of the stress wave as it propagates through the experimental field of view is shown in Fig. 5b, where data has been translated such that y axis represents the striker-gel interface. The first two profiles show the stress wave entering the sample; the final two profiles show the fully developed velocity field increasing approximately linearly up to the striker velocity of $27 \mathrm{~m} / \mathrm{s}$. In the absence of voids, there is no significant change in the wave slope through the field of view of the experiment. 


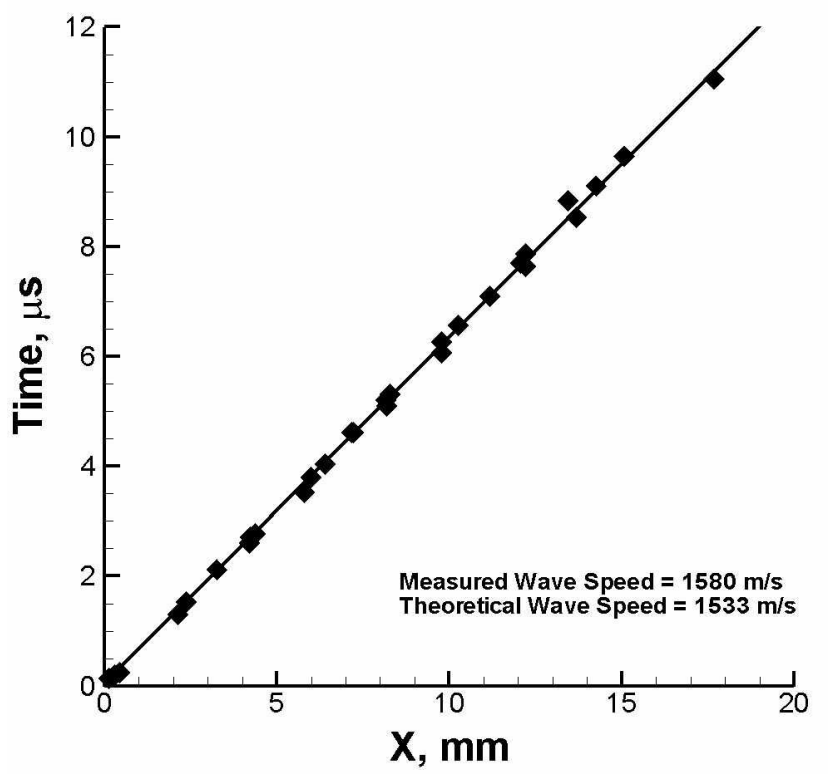

Figure 4: High-speed shadowgraph $x-t$ data of pressure pulse resulting from the elastic wave incident on the striker-sample interface.

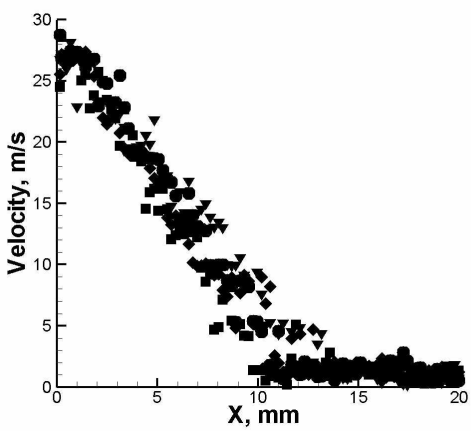

(a)

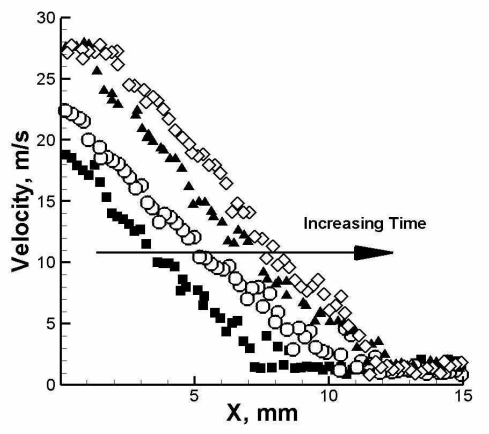

(b)

Figure 5: (a) Velocity profiles through the Agarose-GGB mixture measured at several cross-sections in a single PIV image and (b) evolution of centerline velocity as the stress wave propagates through the field of view. The gel-striker interface is located at $\mathrm{x}=0 \mathrm{~mm}$. Flow is from left to right. 


\section{IV.B. Collapse of a Single Void}

We examine a single void using high-speed shadowgraph images to obtain a time-resolved visualization of the collapse. These data subsequently serve as a time reference in the analysis of the single-shot PIV images and select images are shown in Fig. 6, which also contains simulated schlieren. We calculate the ratio of post-wave pressure to initial pressure $p / p_{o}$ to be 421 , which is intermediate to the weak $\left(p / p_{o}<300\right)$ and strong $\left(p / p_{o}>5000\right)$ regimes defined for shock-induced collapse. ${ }^{12}$ Qualitatively, the void undergoes the asymmetric collapse observed in the strong shock regime, with a high speed jet evident in both the simulated and experimental images. The times shown are the elapsed times referenced from Figs. 6a and 6 f. The arrival of the precursor pulse at the proximal interface of the void is seen in Fig. 6b. Significant wave front curvature can be observed, however this is assumed to have negligible effect, as the precursor pulse does not trigger the void collapse. Instead, as will be seen in the PIV data, the void begins to collapse with the arrival of the stress wave. The genesis of the jet is visible in Fig. 6c, becoming fully developed in Figs. 6d and 6i. Figures 6e and $6 \mathrm{j}$ show the consequence of the jet impingement on the distal interface of the void. A forward-facing, semi-circular pulse propagates into the downstream material in the experimental data, and fully circular pulse is seen in the numerical results.

The experimental time to minimum diameter (i.e. collapse time based upon the jet reaching the distal interface) $t_{D, \min }$ is found to be $24 \mu \mathrm{s} \pm 1.5 \mu \mathrm{s}$. It should be noted that this is not the same as the time to reach minimum volume $t_{V, \text { min }}$. For the single void experimental data, $t_{V, \text { min }}$ is $25.5 \mu \mathrm{s} \pm 1.5 \mu \mathrm{s}$. The collapse times computed from numerical simulations, $t_{D, \min }=22 \mu \mathrm{s}$ and $t_{V, \min }=23 \mu \mathrm{s}$ also compare reasonably well with the corresponding values.

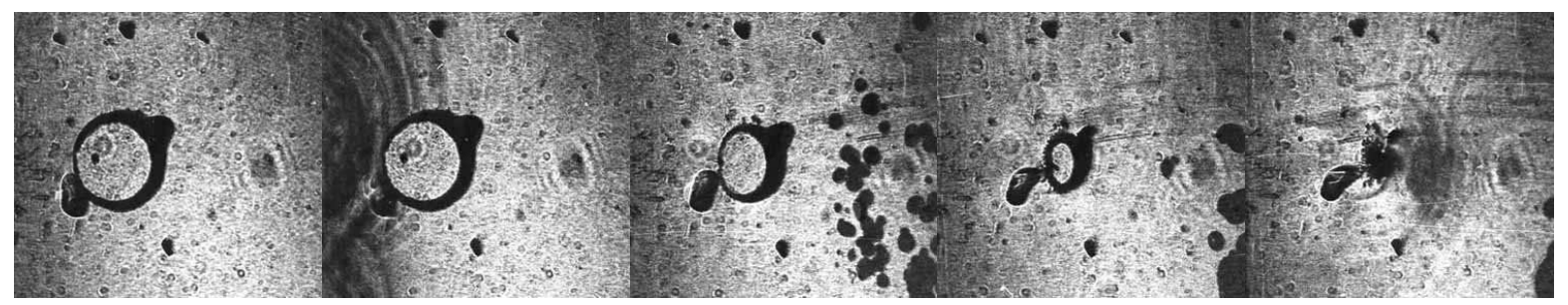

(a) $0 \mu \mathrm{s}$

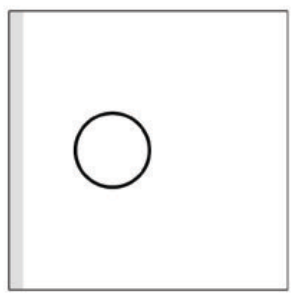

(f) $0 \mu \mathrm{s}$ (b) $4.5 \mu \mathrm{s}$

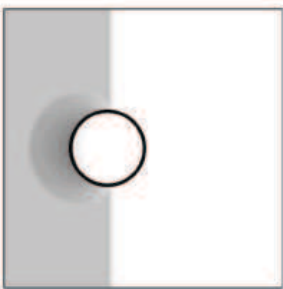

(g) $4.5 \mu \mathrm{s}$ (c) $18 \mu \mathrm{s}$

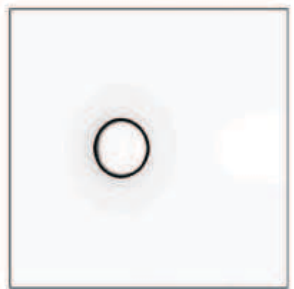

(h) $18 \mu \mathrm{s}$ (d) $25.5 \mu \mathrm{s}$

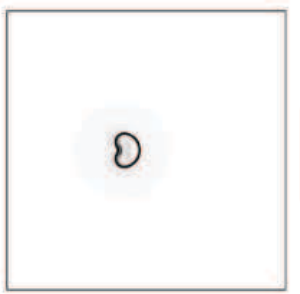

(i) $25.5 \mu \mathrm{s}$ (e) $33 \mu \mathrm{s}$

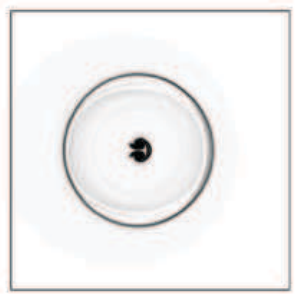

(j) $33 \mu \mathrm{s}$

Figure 6: High speed shadowgraph images, (a)-(e), of a single void collapse. Images are obtained using a laser light source through a gelatinous material; some laser diffraction could not be eliminated. Initial void diameter is $3 \mathrm{~mm}$. Simulated schlieren images, (f)-(j), are shown from numerical simulations at the corresponding times.

The normalized cavity centerline cross-sectional diameter vs time and the normalized cavity internal volume vs time based on cavity interface location are reported in Fig. 7a and Fig. 7b respectively for both experiment and simulation. The centerline diameter versus time plot shows an initial period of nonlinear collapse up to $t / t_{D, \min } \approx 0.25$ in the experimental data. The simulation does not exhibit a linear collapse behavior until a much later time, $t / t_{D, \min } \approx 0.90$. In both simulation and experiment, the distal interface is stationary while the proximal accelerates. The subsequent region appears linear until the jet reaches the distal interface. The cavity volume versus time exhibits similar behavior at the beginning of the collapse. Again, acceleration is observed in the beginning stages, however the nonlinear portion extends only up to $t / t_{V, \min } \approx$ 0.12 in the experimental data and $t / t_{V, \text { min }} \approx 0.55$ in the simulations. Toward the end of the collapse, starting at $t / t_{V, \min } \approx 0.82$ in the experimental data and $t / t_{V, \min } \approx 0.90$ in the simulations, a nonlinear (deceleration) of the volume versus time is observed, potentially due to the increased gas compression within the void. The correlation between the data and simulation in Figs $7 \mathrm{a}$ and $7 \mathrm{~b}$ is good given that the spatial error bars on 
experimental data are $\sim \pm 8 \%$ the initial diameter and the viscosity and elastoplastic behavior of agaroseGGB are not accounted for in the numerical calculations.

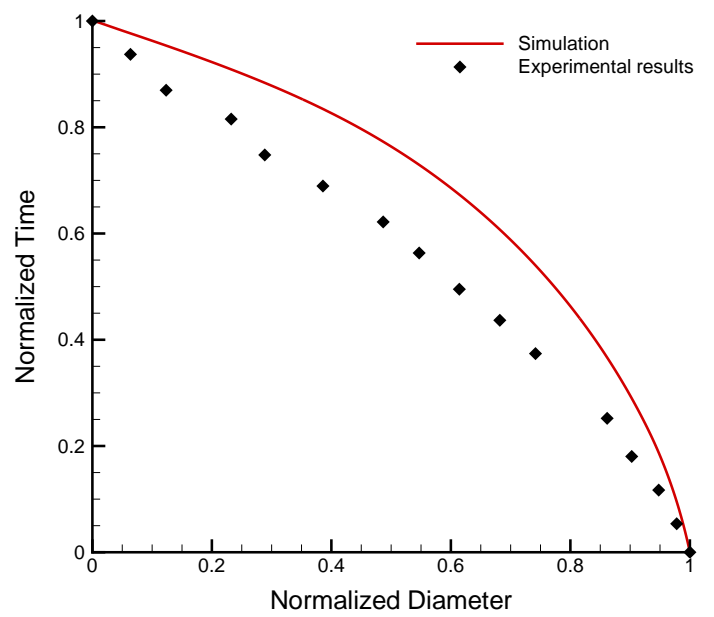

(a)

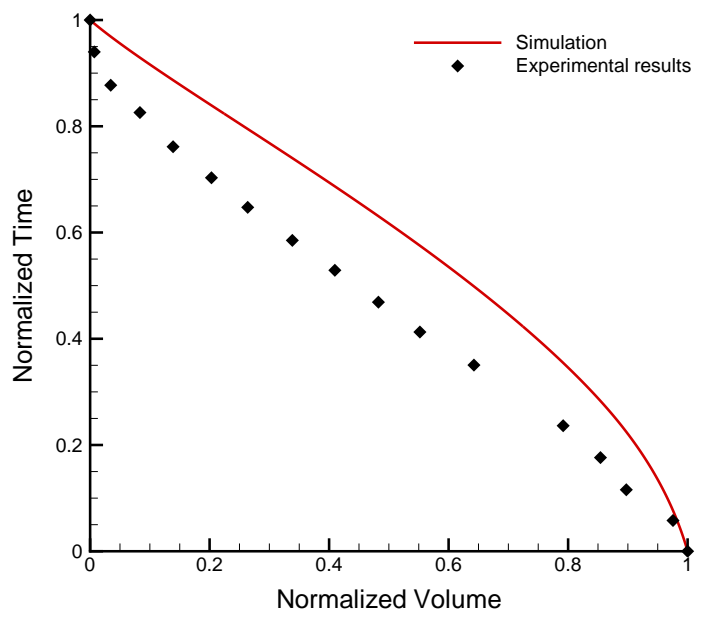

(b)

Figure 7: Normalized a) centerline diameter and b) internal volume versus time for a single void collapse.

PIV measurements are made at selected times after projectile impact, yielding the planar velocity fields in Fig. 8. The blue boundary represents the perimeter of the void during the first PIV frame; the red boundary represents the perimeter of the void during the second PIV frame. The black dotted boundary represents the initial size and location of the void and the white fill region represents the uncollapsed area of the void after the second frame. During the early stages of collapse, there is a region upstream of the void where the velocity is significantly greater than in the free stream, Fig. 8a. The velocity approaches more than three times the post-wave particle velocity. The stress wave front diffracts around the void, and corresponding velocity vector field divergence above and below the void, with a shielded region of zero velocity just downstream of the void, is observed in the PIV images, Fig.s 8a and 8b. In the final image obtained during the cavity collapse, Fig. 8c, the consequence of the wave generated by jet impingement on the distal void interface observed in shadowgraph data, is now observed through velocity data. Radial velocity vectors appear in the previously shielded downstream region of the void, with a profile similar to that of the wave seen in Fig. 6e. The wave appears to advance ahead of the initial stress wave propagating though the material.

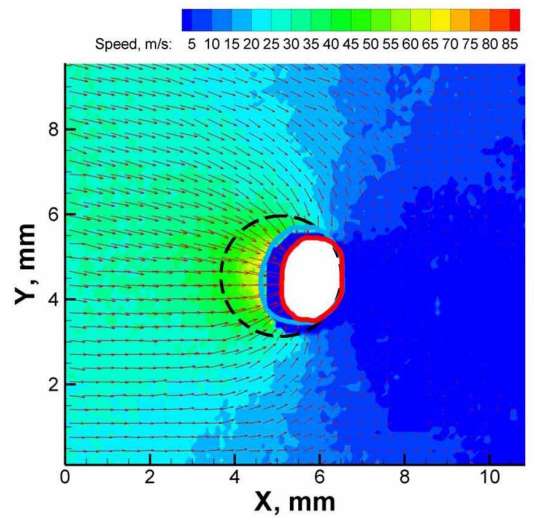

(a) $18 \mu \mathrm{s}$

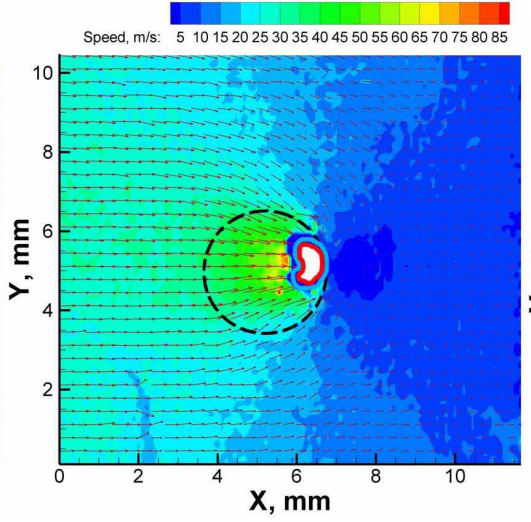

(b) $27 \mu \mathrm{s}$

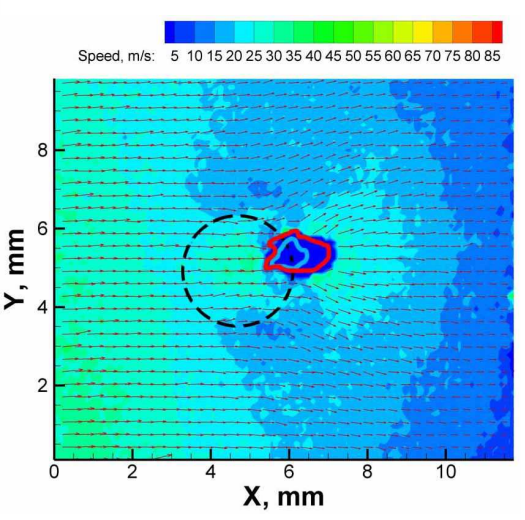

(c) $34.5 \mu \mathrm{s}$

Figure 8: Velocity contours for a single void collapse. Times are referenced from the same scale as in Fig. 6. Initial void diameter is $3 \mathrm{~mm}$. 


\section{IV.C. Single Void Region of Influence}

Dear \& Field ${ }^{32}$ report that cavities having centers up to two diameters apart experience some degree of interaction. We use PIV data to provide a quantitative measure of the velocity induced by a single void collapse, examining the vertical velocity component. These plots reveal two circular regions above and below the void, and is shown in Fig. 9 at different time delays after the beginning of the collapse. During the initial stages of collapse, the surrounding material is accelerated toward the cavity at velocities of up to $40 \mathrm{~m} / \mathrm{s}$, approximately 1.5 times the projectile velocity. After the proximal interface has impacted the distal interface, radial velocity vectors are observed downstream of the void.

We define the region of influence as the area where the induced vertical component of the velocity is greater than $5 \%$ of the free stream velocity. Measurements indicate that void will influence material roughly 1.8 to 1.9 diameters away from the center of the void. As a consequence, the velocity fields surrounding two voids will interact when their centers are double this distance apart (3.6 to 3.8 void diameters).

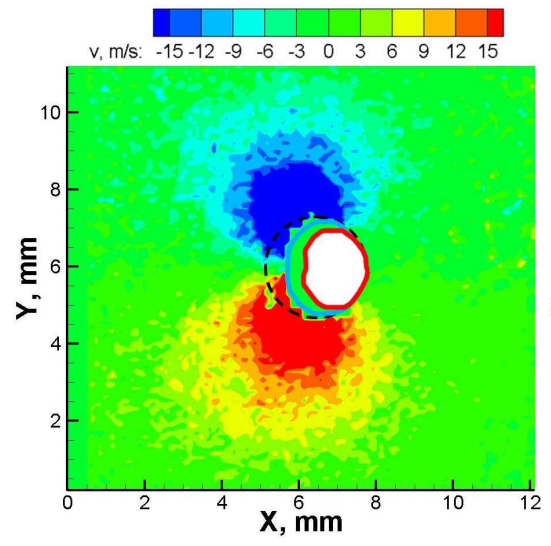

(a) $16.5 \mu \mathrm{s}$

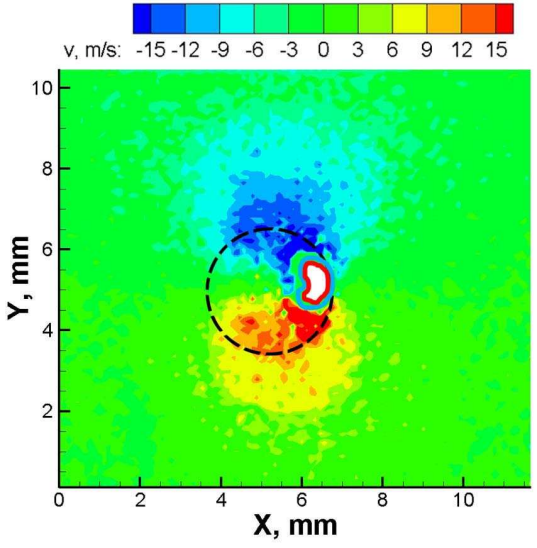

(b) $27 \mu \mathrm{s}$

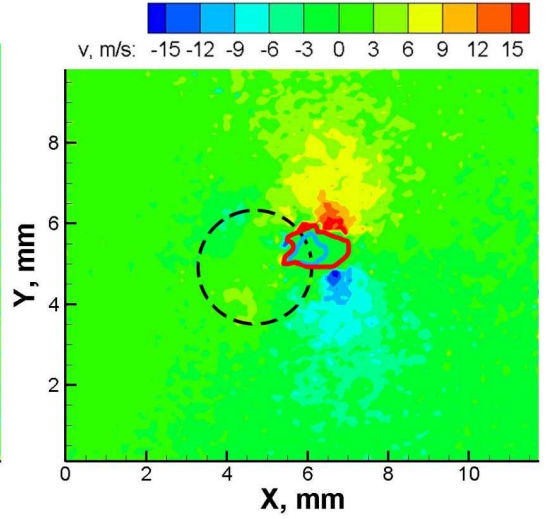

(c) $34.5 \mu \mathrm{s}$

Figure 9: Examples of the velocity field induced by a collapsing void at selected times during the collapse. Times are referenced as in Fig. 6. Initial void diameter is $3 \mathrm{~mm}$. Velocity contours in the range $\pm 15 \mathrm{~m} / \mathrm{s}$ only are shown to emphasize the shape and extent of the region of influence.

\section{IV.D. Comparison of Stress Wave and Shock Wave Loading}

In the present study, we examine the interaction of a stress wave, such as might result from an accidental mechanical insult, with a collapsing cavity. In contrast to a shock wave, the stress wave profile has a length scale that may be comparable to the void diameter. We compare the internal volume history obtained in the present experiments under stress wave loading with literature data for shock-induced cavity collapse, Fig. 10. Bourne \& Field ${ }^{13}$ experimentally investigated shock-induced collapse of cylindrical, air-filled voids in gelatine, with cavity diameters from 3 to $12 \mathrm{~mm}$ and a shock pressure ratio of $0.26 \mathrm{GPa}$. The experimental data from Haas \& Sturtevant ${ }^{33}$ who examined shock collapse of cylindrical helium and Refrigerant 22 gas bubbles in air is also shown. Bourne \& Field ${ }^{13}$ find a linear curve fit to their data over the entire collapse regime. The present data however show linear behavior occurs only over a portion of the collapse, with nonlinear regions at the beginning and end of the collapse history.

The present experimental data are also compared with numerical collapse histories, Fig. 11. Ball et al. ${ }^{15}$ carried out simulations of the Bourne \& Field ${ }^{13}$ shock-induced collapse experiments and report initial and final nonlinear regions. These numerical data show very good agreement with the present experiments over the entire collapse history, with the nonlinear initial and final stages captured in both the experiments and simulations. Ball et al. ${ }^{15}$ report a linear collapse region between $0.27<t / t_{V, \min }<0.81$, which agrees well with the current results. Also shown for reference are data from simulations by Johnsen \& Colonius ${ }^{18}$ of shock-induced collapse of a spherical air bubble in water. Initial acceleration and final deceleration are also observed in these three-dimensional simulations. As expected, the initial volume reduction is slower and the final volume reduction is faster in the three-dimensional case than in the two-dimensional case. 


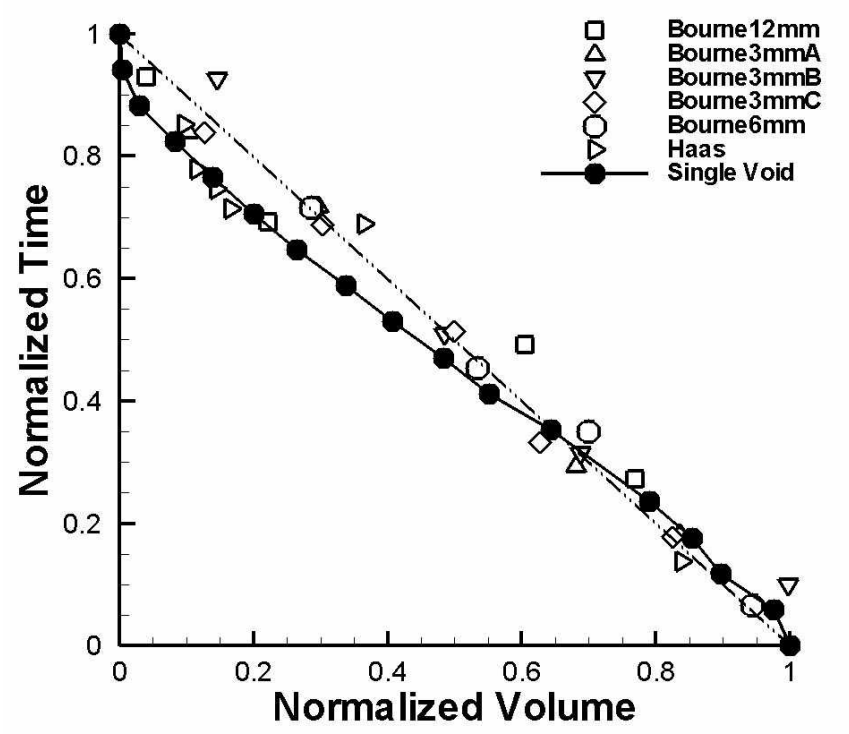

Figure 10: Plots of normalized volume versus normalized time. Collapse time is normalized by the initial void diameter and fluid speed of sound. Current data (Single Void) are compared with experiments of Bourne \& Field ${ }^{13}$ and Haas \& Sturtevant. ${ }^{33}$ (A connecting line between current data points is shown for clarity.) The linear curve fit of Bourne \& Field ${ }^{13}$ is also included.

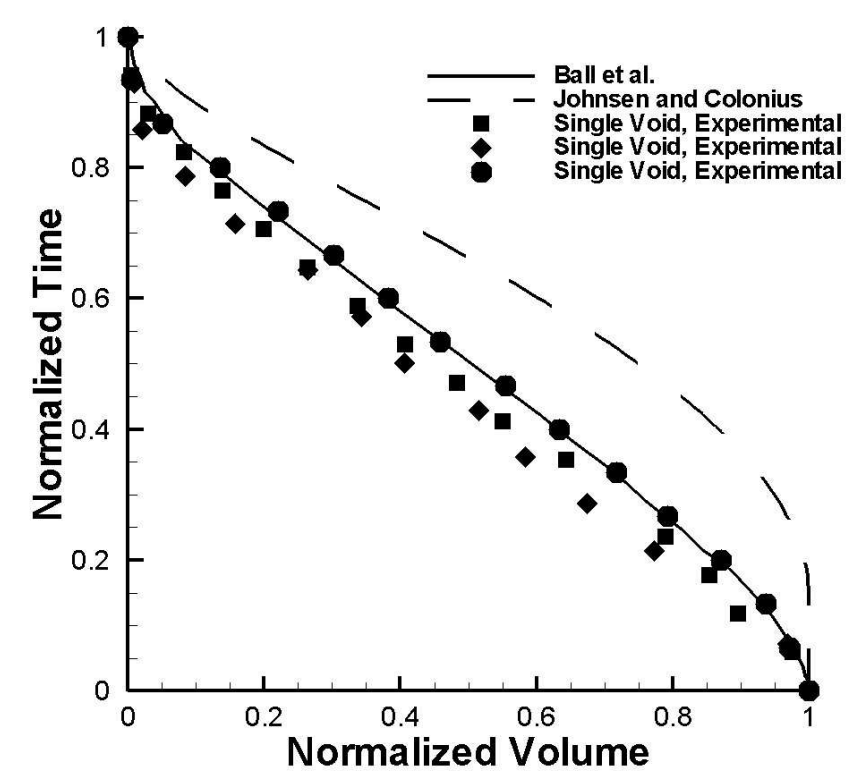

Figure 11: Plots of normalized volume versus normalized time for current experimental data and literature simulations from Ball et al. ${ }^{15}$ (two-dimensional) and Johnsen \& Colonius ${ }^{18}$ (three-dimensional). Collapse time is normalized by the initial void diameter and fluid speed of sound. Good agreement is obtained between experiments and two-dimensional numerical collapse histories. 
The Rayleigh collapse time $t_{c}$ of symmetrical, spherical bubble scales with the pressure difference. ${ }^{34,35}$

$$
t_{c} \sim 0.915\left(\frac{\rho}{p_{\infty}-p_{v}}\right)^{0.5} R_{i}
$$

where $\rho$ is the density in the surrounding medium, $p_{\infty}$ is the pressure at infinity, $p_{v}$ is the internal pressure, and $R_{i}$ is the initial bubble radius. A correction for asymmetric collapse (near a wall) introduced the distance to the wall as a parameter but retained the same pressure and initial bubble radius dependence. ${ }^{36}$ For cylindrical bubbles, no analogue to the Rayleigh equation exists. Small and large amplitude oscillations can be modeled using a wave equation, ${ }^{37}$ but to the authors' knowledge, no collapse time prediction model is available.

We compare the time to minimum volume $t_{V, \min }$ from the present study to a compilation of cylindrical bubble collapse times from the literature as a function of the pressure ratio $p / p_{o}$ across the loading wave, Fig. 12. $t_{V, \text { min }}$ is non-dimensionalized by the initial bubble radius $R_{i}$ and the fluid sound speed $c$.

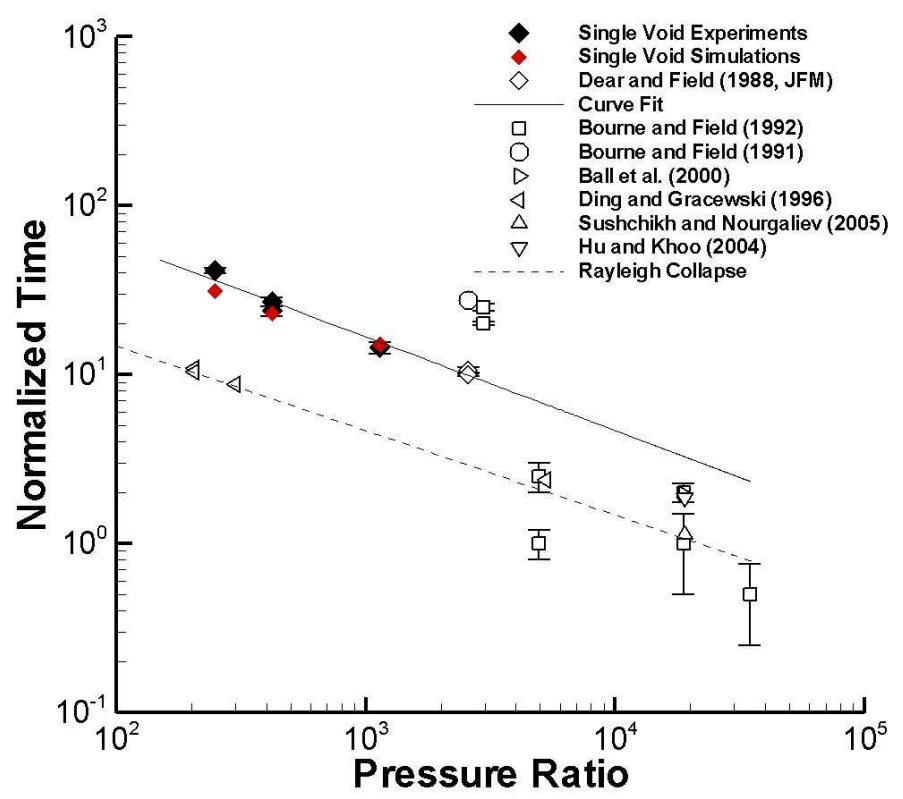

Figure 12: Nondimensionalized collapse time versus loading wave pressure ratio for single, cylindrical voids. A power law curve fit, $t_{V, \min } c / R_{i}=710\left(p / p_{o}\right)^{-0.55}$, is shown to the current data $(\diamond)$ and Dear \& Field ${ }^{32}(\diamond)$.

To achieve a range of loading wave pressure ratios, shadowgraph movie collapse time measurements were made with projectile velocities of 16,27 , and $78 \mathrm{~m} / \mathrm{s}$, corresponding to calculated pressure ratios of 249 , 421, and 1140. Simulations are performed at these conditions as well. At the higher pressure ratios the simulations accurately predict the collapse time of the voids, however there is significant deviation at the lowest pressure ratio. This, again, may be a result of the inviscid calculations, as viscous forces become important at low Reynolds numbers.

A power law curve fit to the current data is shown. Additionally, a power law fit for only the current simulations yields an exponent of -0.48 and for the current experiments, simulations and the data of Dear \& Field ${ }^{32}$ yields an exponent of -0.52. Perhaps surprisingly, these exponents are close to the -0.5 dependence of the Rayleigh curve. Data from the two-dimensional simulations of Ding \& Gracewski, ${ }^{12}$ Ball et al. ${ }^{15} \mathrm{Hu} \&$ Khoo, ${ }^{38}$ and Suschikh \& Nourgaliev ${ }^{39}$ for shock loading conditions are shown. Ding and Gracewski ${ }^{12}$ identify a time of maximum pressure for symmetric collapse, and a time of jet impingement for asymmetric collapse. Ball et al. ${ }^{15} \mathrm{Hu} \&$ Khoo, ${ }^{38}$ and Suschikh \& Nourgaliev, ${ }^{39}$ report a time to minimum volume. While the internal volume histories are very similar to current results, Fig. 11, the collapse times are significantly different. Experiments show increased collapse times over the numerical results. Data from the two-dimensional simulations of Ding and Gracewski ${ }^{12}$ have pressure dependence close to the three-dimensional Rayleigh curve for gas bubbles in water.

The experimental data of Dear \& Field, ${ }^{32}$ Bourne \& Field, ${ }^{40}$ and Bourne \& Field ${ }^{13}$ for cylindrical voids under shock loading are also presented. In spite of the similarity in the experimental setup, there is not good 
agreement between these data and the present study in all cases. The data from Dear \& Field ${ }^{32}$ appear consistent with our data and are included in the curve fit. However, collapse times from the related studies of Bourne and Field are significantly different.

\section{Conclusions}

We investigate the phenomenon of collapsing cavities under stress wave loading, such as occurs in energetic materials in accident scenarios with low impact velocity mechanical insult. Hotspot formation and detonation initiation involves complex thermochemical, fluid and structural processes over an extremely broad range of scales. We study a model problem, both experimentally and numerically, with a single cylindrical void in a gelatinous material. High speed shadowgraph movies provide time-resolved visualizations of the collapse process, in tandem with particle image velocimetry measurements in the surrounding media at selected stages during the collapse. Inviscid two dimensional simulations are carried out at experimental conditions.

We observe asymmetric collapse and the formation of a central internal jet. The high speed jet impinges upon the stationary distal void interface and generates a pressure pulse followed by a radially expanding velocity field. Neighboring voids have been observed to interact when located approximately one diameter apart in previous studies. We quantify a void collapse region of influence by measuring the extent of the vertical velocity field as a function of time.

Internal volume and centerline diameter histories during the collapse are reported. In contrast to the entirely linear behavior reported in previous experiments, the present internal volume versus time data show an initial acceleration of the proximal interface, followed by linear collapse, and a final deceleration. Very good agreement is obtained with two-dimensional numerical simulations from the literature which also capture the nonlinear regions in the collapse history. Current simulations also exhibit distinct linear and non-linear regions as well. Centerline diameter data show an initial acceleration followed by a linear regime, but no final deceleration due to the formation of the central jet. The experimental volume history data for the single void under stress wave loading are in reasonable agreement with current and existing numerical simulations of a void under shock wave loading, indicating the pressure ratio is the dominant parameter in determining the collapse dynamics. It is important to note that during the final stages of the collapse process in many numerical simulations, results are largely influenced by the inherent numerical diffusion of shock capturing methods. Interfaces will spatially diffuse, causing volume/diameter calculations to become very inaccurate, especially when interfaces are very near to each other.

For a spherical bubble, the Rayleigh collapse time scales with the square root of the pressure difference. No direct analogue for the Rayleigh-Plesset equation exists for cylindrical bubbles. We compare time to minimum volume results from the present study with existing numerical and current experimental and computational data. Normalized collapse time is generally found to increase with decreasing pressure ratio. A power law can be fit to the experimental data from the present study and from Dear \& Field, ${ }^{32}$ and an exponent of -0.55 is obtained. Current experiments and simulations exhibit good collapse time agreement at higher pressure ratios, but deviate at the lowest pressure ratio. Experimental data from the related studies of Bourne \& Field ${ }^{13}$ do not fall on this curve. Simulations for shock-induced collapse do not take into account the ramped profile and therefore universally underpredict experimental collapse times but appear to show a similar pressure dependence as experiments.

\section{Acknowledgments}

The authors gratefully acknowledge Prof John Lambros for the generous loan of laboratory equipment and space which made this study possible. We thank Matthew Parker for his initial experiments, Prof Jonathan Freund, Prof Carlos Pantano, and Prof Scott Stewart for useful discussions comparing experiments and simulations, and Prof Greg Elliott for helpful input with PIV measurements. We also thank Dr Eric Johnsen and Prof Tim Colonius for sharing their numerical data. This work was supported in part by the US Department of Energy through the University of California under subcontract B523819.

\section{References}

${ }^{1}$ Bowden, F. P. and Yoffe, A. D., Ignition and Growth of Explosion in Liquids and Solids, Cambridge University Press, Cambridge, UK, 1952. 
${ }^{2}$ Tarver, C. M., Chidester, S. K., and Nichols, A. L., "Critical Conditions for Impact- and Shock-Induced Hot Spots in Solid Explosives," Journal of Physical Chemistry, Vol. 100, 1996, pp. 5794-5799.

${ }^{3}$ Mader, C. L., "Initiation of detonation by the interaction of shocks and density discontinuities," Physics of Fluids, Vol. 8, 1965, pp. 1811-1816.

${ }^{4}$ Carroll, M. M. and Holt, A. C., "Static and dynamic pore-collapse relations for ductile porous materials," Journal of Applied Physics, Vol. 43, No. 4, 1972, pp. 1626-1636.

${ }^{5}$ Khasainov, B. A., Borisov, A. A., Ermolaev, B. S., and Korotkov, A. I., "Two phase visco-plastic model of shock initiation of detonation in high density pressed explosives," Proceedings of the "th Symposium (International) on Detonation, Annapolis, MN, 1981.

${ }^{6}$ Maiden, D. E. and Nutt, G. L., "A hot-spot model for calculating the threshold for shock initiation of pyrotechnic mixtures," 11th International Pyrotechnics Seminar, Vail, CO, 1986, pp. 813-826.

${ }^{7}$ Bourne, N. K. and Field, J. E., "Explosive ignition by the collapse of cavities," Proceedings of the Royal Society A, Vol. 455, 1999, pp. 2411-2426.

${ }^{8}$ Kang, J., Butler, P., and Baer, M., "A thermochemical analysis of hot spot formation in condensed-phase, energetic materials," Combustion and Flame, Vol. 89, 1992, pp. 117-139.

${ }^{9}$ Menikoff, R., "Pore collapse and hot spots in HMX," American Physical Society Topical Conference, Shock Compression of Condensed Matter, 2003, (also LA-UR-03-3113), Portand, OR.

${ }^{10}$ Tran, L. and Udaykumar, H., "Simulation of void collapse in an energetic material, Part 1: Inert case," Journal of Propulsion and Power, Vol. 22, No. 5, 2006, pp. 947-958.

${ }^{11}$ Dear, J. P., Field, J. E., and Walton, A. J., "Gas compression and jet formation in cavities collapsed by a shock wave," Nature, Vol. 332, 1988, pp. 505-508.

${ }^{12}$ Ding, Z. and Gracewski, S. M., "The behaviour of a gas cavity impacted by a weak or strong shock wave," Journal of Fluid Mechanics, Vol. 309, 1996, pp. 183-209.

${ }^{13}$ Bourne, N. K. and Field, J. E., "Shock-induced collapse of single cavities in liquids," Journal of Fluid Mechanics, Vol. 244, 1992, pp. 225-240.

${ }^{14}$ Bourne, N. K. and Field, J. E., "Collapsing cavities in reactive and non-reactive media," 19th International Congress on High-Speed Photography and Photonics SPIE, Vol. 1358, 1990, pp. 1046-1055.

${ }^{15}$ Ball, G. J., Howell, B. P., Leighton, T. G., and Schofield, M. J., "Shock-induced collapse of a cylindrical air cavity in water: a Free-Lagrange simulation," Shock Waves, Vol. 10, 2000, pp. 265-276.

${ }^{16}$ Bourne, N. K. and Milne, A. M., "The temperature of a shock-collapsed cavity," Proceedings of the Royal Society A, Vol. 459, 2003, pp. 1851-1861.

${ }^{17}$ Turangan, C. K., Jamaluddin, A. R., Ball, G. J., and Leighton, T. G., "Free-Lagrange simulations of the expansion and jetting collapse of air bubbles in water," Journal of Fluid Mechanics, Vol. 598, 2008, pp. 1-25.

${ }^{18}$ Johnsen, E. and Colonius, T., "Numerical simulations of non-spherical bubble collapse," Journal of Fluid Mechanics (to appear), Vol. 629, 2009, pp. 231-262.

${ }^{19}$ Swantek, A. B. and Austin, J. M., "Collapse of void arrays under stress wave loading," Journal of Fluid Mechanics (to appear), 2010.

${ }^{20}$ Kodama, T. and Tomita, Y., "Cavitation bubble behavior and bubble-shock wave interaction near a gelatin surface as a study of in vivo bubble dynamics," Journal of Applied Physics B, Vol. 70, 2000, pp. 139-149.

${ }^{21}$ Murphy, M. J., Adrian, R. J., Stewart, D. S., Elliott, G. S., Thomas, K. A., and Kennedy, J. E., "Visualization of Blast waves created by exploding bridge wires," Journal of Visualization, Vol. 8, No. 2, 2005, pp. 125-135.

${ }^{22}$ Murphy, M. J. and Adrian, R. J., "Particle response to shock waves in solids: dynamic witness plate/PIV method for detonations," Experiments in Fluids, Vol. 43, 2007, pp. 163-171.

${ }^{23}$ LeVeque, R. J., Finite Volume Methods for Hyperbolic Problems, Cambridge University Press, New York, 2002.

${ }^{24}$ Toro, E. F., Spruce, M., and Speares, W., "Restoration of the contact surface in the HLL-Riemann solver," Shock Waves, Vol. 4, No. 1, 1994, pp. 25-34.

${ }^{25}$ Olsson, E., Kreiss, G., and Zahedi, S., "A conservative level set method for two phase flow II," Journal of Computational Physics, Vol. 225, No. 1, 2007, pp. 785-807.

${ }^{26}$ Sun, Y. and Beckermann, C., "Sharp interface tracking using the phase-field equation," Journal of Computational Physics, Vol. 220, No. 2, 2007, pp. 626-653.

${ }^{27}$ Shukla, R. K., Pantano, C., and Freund, J. B., "An interface capturing method for simulation of multiphase compressible flows," submitted, 2009.

${ }^{28}$ Gottlieb, S. and Shu, C. W., "Total variation diminishing Runge-Kutta schemes," Mathematics of Computation, Vol. 67, 1998 , pp. 73-85.

${ }^{29}$ Jaramillo, E., Sewell, T. D., and Strachan, A., "Atomic-level view of inelastic deformation in shock loaded molecular crystal," Physical Review B, Vol. 76, 2007, pp. 6.

${ }^{30}$ Menikoff, R., "Notes on Elastic-plastic flow," Tech. rep., LA-UR-03-0047, Los Alamos National Laboratories, Los Alamos, NM, 2003

${ }^{31}$ Dick, J. J., Hooks, D. E., Menikoff, R., and Martinez, A. R., "Elastic-plastic wave profiles in cyclotetramethylene tetranitramine crystals," Journal of Applied Physics, Vol. 96, No. 1, 2004, pp. 374-379.

${ }^{32}$ Dear, J. P. and Field, J. E., "A study of the collapse of arrays of cavities," Journal of Fluid Mechanics, Vol. 190, 1988, pp. 409-425.

${ }^{33}$ Haas, J. F. and Sturtevant, B., "Interaction of weak shock waves with cylindrical and spherical gas inhomogeneities," Journal of Fluid Mechanics, Vol. 181, 1987, pp. 41-76.

${ }^{34}$ Rayleigh, L., "On the pressure developed in a liquid during the collapse of a spherical cavity," Philosophical Magazine, Vol. 34, 1917, pp. 94-98. 
${ }^{35}$ Brennan, C. E., Fundamentals of Multi-Phase Flow, Cambridge University Press, Cambridge, UK, 2005.

${ }^{36}$ Rattray, M., Perturbation effects in cavitation bubble dynamics, Ph.D. thesis, California Institute of Technology, Pasadena, California, 1951.

${ }^{37}$ Epstein, D. and Keller, J. B., "Expansion and contraction of planar, cylindrical, and spherical underwater gas bubbles," Journal of the Acoustical Society of America, Vol. 52, No. 3, 1972, pp. 975.

${ }^{38} \mathrm{Hu}, \mathrm{X}$. Y. and Khoo, B. C., "An interface interaction method for compressible multifluids," Journal of Computational Physics, Vol. 198, No. 1, 2004, pp. 35-64.

${ }^{39}$ Sushchikh, S. Y. and Nourgaliev, R. R., "Shock Waves and Flow Patterns in Shock-Induced Bubble Collapse," 43rd AIAA Aerospace Sciences Meeting and Exhibit, , No. Paper no. 2005-1291, 2005, Reno, NV.

${ }^{40}$ Bourne, N. K. and Field, J. E., "Bubble Collapse and the Initiation of Explosion," Proceedings of Mathematical and Physical Sciences, Vol. 435, No. 1894, 1991, pp. 423-435. 